\title{
Diaz-Metcalf and Pólya-Szegő type inequalities associated with Saigo fractional integral operator
}

\author{
Ram K. Saxena ${ }^{a}$, Jitendra Daiya ${ }^{a}$, Tibor K. Pogány ${ }^{b, \star}$ \\ a Department of Mathematics and Statistics, Jai Narain Vyas University, Jodhpur, India \\ ${ }^{b}$ Faculty of Maritime Studies, University of Rijeka, Rijeka 51000, Croatia \\ ${ }^{\star}$ Corresponding Author \\ E-mail: ram.saxena@yahoo.com, jitendra.daiya25@gmail.com, poganj@brod.pfri.hr
}

\begin{abstract}
This paper deals with the derivation of certain new Pólya-Szegő type inequalities by making use of the Saigo fractional integral operator. The results obtained cover the same kind of conclusions in the case of Riemann-Liouville and Erdélyi-Kober fractional integral operators.
\end{abstract}

2010 Mathematics Subject Classification. 26A33. 26D10, 26D15

Keywords. Diaz-Metcalf inequality, Pólya-Szegő inequality, Saigo fractional integral operator.

\section{Introduction and results required}

The authors of this research note realized recently a study [12] in which the cornerstone was the Saigo-type fractional integral [11] applied to some suitably bounded and/or integrable functions. Following the study on Saigo type fractional integral operator, firstly we shall derive certain new integral inequalities related to Diaz-Metcalf and Pólya-Szegő inequalities in Saigo type fractional integral setting together with related special cases which turn out to be the widely known classical inequalities by Rennie and Schweitzer.

The main building-block in both stories is the integral mean of a suitable input function $h$ on a finite interval $[a, b]$ defined by

$$
\mathscr{M}(h)=\frac{1}{b-a} \int_{a}^{b} h(x) \mathrm{d} x .
$$

Its further specialized shapes adopted to the situations occur in the sequel.

The symbol $\chi_{S}(t)$ stands for the characteristic function of the set $S, \delta_{\lambda \mu}$ is the Kronecker symbol, while under $L_{\varphi}^{p}[A], p \in \mathbb{R}$ we mean the function space $\left\{\left.h\left|\int_{A}\right| h(t)\right|^{p} \varphi(t) d t<\infty\right\}$.

\subsection{Diaz - Metcalf weighted integral inequality.}

The article [3] where the inequality initially appeared and e.g. the celebrated monograph [7] contain this classical result, also see [8] for the probabilistic point of view and equality analysis.

Let $f, g$ be Borel - functions, such that

$$
m \leq f(x) \leq M, \quad 0<n \leq g(y) \leq N, \quad \text { a.e. }(x, y) \in[a, b] \times[c, d] .
$$

Let $w(x, y)$ be a nonnegative normalized weight function for which $\operatorname{supp}(w)=[a, b] \times[c, d]$, i.e. $\int_{a}^{b} \int_{c}^{d} w(x, y) \mathrm{d} x \mathrm{~d} y=1$ and

$$
\int_{c}^{d} w(x, y) \mathrm{d} y=w_{1}(x), \quad \int_{a}^{b} w(x, y) \mathrm{d} x=w_{2}(y) .
$$

Tbilisi Mathematical Journal 7(2) (2014), pp. 11-20.

Tbilisi Centre for Mathematical Sciences.

Received by the editors: 03 July 2014

Accepted for publication: 13 October 2014 
Introducing the weighted integral mean with the weight $p$ of the function $h$ with a support being either a 1-, or on 2-dimensional rectangle, as the functional

$$
\mathscr{M}_{p}(h):= \begin{cases}\int_{a}^{b} h(x) w_{j}(x) \mathrm{d} x, & p \equiv w_{j}, j=1,2, \\ \int_{a}^{b} \int_{c}^{d} h(x, y) w(x, y) \mathrm{d} x \mathrm{~d} y, & p \equiv w,\end{cases}
$$

the weighted integral Diaz-Metcalf inequality reads

$$
\mathscr{M}_{w_{1}}\left(f^{2}\right)+\frac{m M}{n N} \mathscr{M}_{w_{2}}\left(g^{2}\right) \leq\left(\frac{m}{N}+\frac{M}{n}\right) \mathscr{M}_{w}(f g) .
$$

Here the equality appears iff either (i) $m / N=M / n$ or (ii) $m / N<M / n$ and

$$
\int_{\mathbb{I}_{x, y}} w(x, y) \mathrm{d} x \mathrm{~d} y=1,
$$

where $\mathbb{I}_{x, y}:=\{(x, y): f(x) / g(y) \in\{m / N, M / n\}\}$.

However, the specification $w(x, y)=(b-a)^{-1} \chi_{[a, b]^{2}}(x, y) \delta_{x y}$ infers the classical Diaz - Metcalf equal - weight integral inequality:

$$
\mathscr{M}\left(f^{2}\right)+\frac{m M}{n N} \mathscr{M}\left(g^{2}\right) \leq\left(\frac{m}{N}+\frac{M}{n}\right) \mathscr{M}(f g) .
$$

as $w_{1}(x)=(b-a)^{-1} \chi_{[a, b]}(x)=w_{2}(x)$. The equality in (1.4) holds iff either (i) $m / N=M / n$ or (ii) $m / N<M / n$ and

$$
\int_{\mathbb{I}_{x}} w_{1}(x) d x=1
$$

where $\mathbb{I}_{x} \equiv \mathbb{I}_{x, x}, x \in[a, b]$, see also [3] and [7, p. 64].

\subsection{Pólya - Szegő weighted integral inequality $[7,8]$.}

Let $f, g$ be positive Borel - functions which satisfy (1.1), that is

$$
0<m \leq f(x) \leq M, \quad 0<n \leq g(y) \leq N, \quad \text { a.s. }(x, y) \in[a, b] \times[c, d],
$$

and let $w(x, y)$ be a nonnegative weight function with $\operatorname{supp}(w)=[a, b] \times[c, d]$ for which

$$
\int_{c}^{d} w(x, y) \mathrm{d} y=w_{1}(x), \quad \int_{a}^{b} w(x, y) \mathrm{d} x=w_{2}(y) .
$$

Then it holds

$$
\frac{\mathscr{M}_{w_{1}}\left(f^{2}\right) \mathscr{M}_{w_{2}}\left(g^{2}\right)}{\mathscr{M}_{w}(f g)} \leq \frac{1}{4}\left(\sqrt{\frac{m n}{M N}}+\sqrt{\frac{M N}{m n}}\right)^{2} .
$$

The equality in (1.5) holds iff the following conditions are satisfied: $[a, b] \equiv[c, d]$ and

$$
f(x)=m \chi_{S}(x)+M \chi_{[a, b] \backslash S}(x), \quad g(y)=N \chi_{[a, b] \backslash S}(y)+n \chi_{S}(y),
$$


for a Borel set $S \subseteq[a, b]$ of Lebesgue measure

moreover

$$
|S|=\frac{M n(b-a)}{m N+M n},
$$

$w(x, y)=0, \quad(x, y) \in S^{2} \cup([a, b] \backslash S)^{2}$.

When $[a, b]=[c, d]$ and $w(x, y)=(b-a)^{-1} \chi_{[a, b]^{2}}(x, y) \delta_{x y}$, we get

$$
\frac{\mathscr{M}\left(f^{2}\right) \mathscr{M}\left(g^{2}\right)}{[\mathscr{M}(f g)]^{2}} \leq \frac{1}{4}\left(\sqrt{\frac{m n}{M N}}+\sqrt{\frac{M N}{m n}}\right)^{2} .
$$

When $f, g$ satisfy (1.6) and (1.7), then (1.8) becomes equality and vice versa. The inequality (1.8) originates back to Pólya and Szegő [9, p.81 \& pp.251-252, Problem 93] where, however, no equality analysis was performed.

We point out that Anber and Dahmani [1] obtained certain Pólya-Szegő-type inequalities by making use of Riemann-Liouville fractional integral operator.

Remark 1.1. According to the observation by Diaz and Metcalf [3, p. 417] it is clear that because (1.4) and since

$$
\left(\mathscr{M}^{1 / 2}\left(f^{2}\right)-\sqrt{\frac{m N}{n M}} \mathscr{M}^{1 / 2}\left(f^{2}\right)\right)^{2} \geq 0
$$

we immediately arrive at the Pólya-Szegő inequality (1.8). Modest adoptions of the same arguments lead from the weighted variant (1.3) and the previous relation (1.9) to the weighted Pólya-Szegö inequality (1.5) as well.

\subsection{Fractional integration.}

Following Saigo [11] we recall the definition of a fractional integration operator in the form considered by Saxena et al. [12].

Definition 1.2. Let $\Re\{\alpha\}, \eta>0, \beta \in \mathbb{R}$. The Saigo fractional integral of the function $f$ on $\mathbb{R}_{+}$is defined as

$$
I_{0, t}^{\alpha, \beta, \eta}[f]=\left\{\begin{array}{l}
\frac{t^{-\beta}}{\Gamma(\alpha)} \int_{0}^{1}(1-x)_{2}^{\alpha-1} F_{1}\left[\begin{array}{c}
\alpha+\beta,-\eta \\
\alpha
\end{array} ; 1-x\right] f(t x) \mathrm{d} x \\
\frac{\mathrm{d}^{n}}{\mathrm{~d} t^{n}} I_{0, t}^{\alpha+n, \beta-n, \eta-n}[f] \quad 0<\Re\{\alpha\}+n \leq 1, n \in \mathbb{N},
\end{array}\right.
$$

where ${ }_{2} F_{1}$ stands for the Gaussian hypergeometric function.

The Riemann-Liouville and Erdélyi-Kober fractional integral operators follow as special cases of (1.10), viz.

$$
\begin{array}{ll}
I_{0, t}^{\alpha}[f]:=I_{0, t}^{\alpha,-\alpha, \eta}[f]=\frac{t^{\alpha}}{\Gamma(\alpha)} \int_{0}^{1}(1-x)^{\alpha-1} f(t x) \mathrm{d} x & \Re\{\alpha\}>0, \\
I_{0, t}^{\alpha, \eta}[f]:=I_{0, t}^{\alpha, 0, \eta}[f]=\frac{1}{\Gamma(\alpha)} \int_{0}^{1}(1-x)^{\alpha-1} x^{\eta} f(t x) \mathrm{d} x & \Re\{\alpha\}, \eta>0 .
\end{array}
$$


For further details of Saigo fractional integral operator related with inequalities one can refer to the papers by Saxena et al. [12] and Jankov and Pogány [6].

It is worth mentioning the following result is given by Saxena et al. [12, p. 672, Theorem 2.1]. Let $\eta-\beta>0, \mu>0, \kappa+\alpha>0$. Then there holds the formula

$$
I_{0, t}^{\alpha, \beta, \eta}\left[x^{\mu-1}(t-x)^{\kappa}\right]=\frac{t^{\kappa+\mu-\beta-1} \mathrm{~B}(\mu, \kappa+\alpha)}{\Gamma(\alpha)} \cdot{ }_{3} F_{2}\left[\begin{array}{c}
\alpha+\beta, \kappa+\alpha,-\eta \\
\alpha, \kappa+\alpha+\mu
\end{array} ; 1\right],
$$

where $\mathrm{B}(\cdot, \cdot)$ denotes the familiar Beta-function. When $\mu=1, \kappa=0$, employing [4, p. 806, Eq. $\left.7.512^{4}\right]$ for $\Re(\alpha), \eta>0, \beta<1$, we get

$$
I_{0, t}^{\alpha, \beta, \eta}[1]=\frac{t^{-\beta}}{\Gamma(\alpha+1)}{ }_{2} F_{1}\left[\begin{array}{c}
\alpha+\beta,-\eta \\
\alpha+1
\end{array} ; 1\right]=\frac{\Gamma(\eta+1-\beta) t^{-\beta}}{\Gamma(1-\beta) \Gamma(\alpha+1+\eta)}
$$

also see [12, p. 673, Remark 2.2].

\subsection{On the Saigo-type weighted integral mean.}

The Čebyšev functional for the Saigo type kernel contaning the Gaussian hypergeometric term ${ }_{2} F_{1}$ has been introduced by Saxena et al. [12]. Comparing the weight function $w(x, y)$ (introduced in the section 1.1) to be a multiplicative separable function $w(x, y)=w_{\mathrm{S}}(x) w_{\mathrm{S}}(y),(x, y) \in[0,1]^{2}$, where

$$
w_{\mathrm{S}}(x):=\frac{t^{-\beta}}{\Gamma(\alpha) I_{0, t}^{\alpha, \beta, \eta}[1]}(1-x)^{\alpha-1}{ }_{2} F_{1}\left[\begin{array}{cc}
\alpha+\beta,-\eta & ; 1 \\
\alpha+1 &
\end{array}\right],
$$

the marginal weight becomes $t$-free:

$$
w_{\mathrm{S}}(x)=\frac{\alpha(1-x)^{\alpha-1}}{{ }_{2} F_{1}\left[\begin{array}{cc}
\alpha+\beta,-\eta \\
\alpha+1
\end{array} ; 1\right]}{ }_{2} F_{1}\left[\begin{array}{c}
\alpha+\beta,-\eta \\
\alpha
\end{array} ; 1-x\right] .
$$

The Saigo-type weighted integral mean (compare with (1.2)) of a suitably choosen input function $f$ has been introduced by Saxena et al. [12, p. 673, Definition 2.3] in the form

$$
\mathscr{M}_{\mathrm{S}}^{\eta}(f)=\mathscr{M}_{0, t}^{\alpha, \beta, \eta}(f)=\frac{I_{0, t}^{\alpha, \beta, \eta}[f]}{I_{0, t}^{\alpha, \beta, \eta}[1]}=\int_{0}^{1} w_{\mathrm{S}}(x) f(t x) \mathrm{d} x, \quad t>0,
$$

for all $\alpha, \eta>0, \beta<1$.

Further obvious reductions imply for $\beta=-\alpha$ the Riemann-Liouville fractional integral operator variant of the Diaz-Metcalf inequality, similarly $\beta=0$ gives the Erdélyi-Kober case. In these cases, the associated fractional integral means become

$$
\mathscr{M}_{\mathrm{RL}}(f)=\mathscr{M}_{0, t}^{\alpha,-\alpha, \eta}(f), \quad \mathscr{M}_{\mathrm{EK}}(f)=\mathscr{M}_{0, t}^{\alpha, 0, \eta}(f),
$$

in which the defining weights are:

$$
w_{\mathrm{RL}}(x)=\alpha(1-x)^{\alpha-1}, \quad w_{\mathrm{EK}}(x)=\frac{\Gamma(1+\alpha+\eta)}{\Gamma(\alpha) \Gamma(1+\eta)} x^{\eta}(1-x)^{\alpha-1} .
$$

In the RL and EK cases $\alpha, \eta>0$. 


\section{Diaz-Metcalf inequality for Saigo type operator}

Now we present the Diaz-Metcalf type inequality for Saigo fractional integral.

Theorem 2.1. Let $f, g \in L_{w_{\mathrm{S}}}^{1}[0, t]$ and

$$
0<m \leq f(x) \leq M<\infty, \quad 0<n \leq g(x) \leq N<\infty ; \quad x \in[0, t] .
$$

Then for all $\Re\{\alpha\}>0, \beta<1, \eta>\beta-1$, and for all $t>0$ we have

$$
\mathscr{M}_{\mathrm{S}}\left(f^{2}\right)+\frac{m M}{n N} \mathscr{M}_{\mathrm{S}}\left(g^{2}\right) \leq\left(\frac{m}{N}+\frac{M}{n}\right) \mathscr{M}_{\mathrm{S}}(f g) \quad \Re(t)>0 .
$$

The equality in (2.1) holds iff $m / N=M / n$.

Proof. By the assumptions

$$
\frac{m}{N} \leq \frac{f(t x)}{g(t x)} \leq \frac{M}{n} \quad t>0, x \in[0,1],
$$

which implies

$$
\left(\frac{M}{n} g(t x)-f(t x)\right)\left(f(t x)-\frac{m}{N} g(t x)\right) \geq 0 .
$$

In turn, the expanded inequality

$$
\left(\frac{M}{n}+\frac{m}{N}\right) f(t x) g(t x) \geq f^{2}(t x)+\frac{m M}{n N} g^{2}(t x)
$$

multiplied by the Saigo's hypergeometric kernel

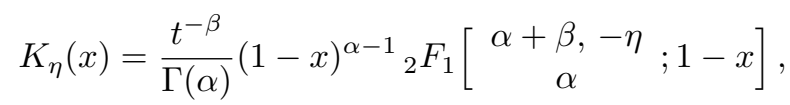

and integrated with respect to $x \in[0,1]$ becomes

$$
I_{0, t}^{\alpha, \beta, \eta}\left[f^{2}\right]+\frac{m M}{n N} I_{0, t}^{\alpha, \beta, \eta}\left[g^{2}\right] \leq\left(\frac{M}{n}+\frac{m}{N}\right) I_{0, t}^{\alpha, \beta, \eta}[f g] .
$$

Dividing it by the normalizing factor $I_{0, t}^{\alpha, \beta, \eta}[1]$ we get the asserted inequality $(2.1)$.

It is not difficult to see that $(2.2)$ is equivalent to

$$
I_{0, t}^{\alpha, \beta, \eta}\left[\left(f-\sqrt{\frac{m M}{n N}} g\right)^{2}+2\left\{\sqrt{\frac{m M}{n N}}-\frac{1}{2}\left(\frac{m}{N}+\frac{n}{M}\right)\right\} f g\right] \leq 0 .
$$

Now, having in mind the Arithmetic mean-Geometric mean inequality, the equality analysis follows; see the end of the subsection 1.2. and also [3] and [7, p. 64].

Q.E.D.

Now, to obtain related Diaz-Metcalf like inequalities it is enough in (2.1) to specify $\beta=-\alpha$ and $\beta=0$ respectively. However, we skip to expose these special cases.

Now, we give a modest extension of the Diaz-Metcalf inequality (2.1) considering two different weighted Saigo integral means which contain arbitrary independent scale parameters $\eta$ and $\xi$. In the sequel we will denote

$$
\mathscr{M}_{\mathrm{S}}^{\eta}(f):=\mathscr{M}_{0, t}^{\alpha, \beta, \eta}(f)
$$


Theorem 2.2. Let $f, g$ be integrable functions on $\mathbb{R}_{+}$which satisfy (1.1). Then for all $\Re\{\alpha\}>$ $0, \beta<1 ; \eta, \xi \in \mathbb{R}$ satisfying $\min \{\eta, \xi\}>\beta-1$ we have

$$
\mathscr{M}_{\mathrm{S}}^{\eta}\left(f^{2}\right)+\frac{m M}{n N} \mathscr{M}_{\mathrm{S}}^{\xi}\left(g^{2}\right) \leq\left(\frac{m}{N}+\frac{M}{n}\right) \mathscr{M}_{\mathrm{S}}^{\eta}(f) \mathscr{M}_{\mathrm{S}}^{\xi}(g) \quad \Re(t)>0 .
$$

Proof. By the assumption (1.1) we obtain

$$
\left(\frac{M}{n} g(t y)-f(t x)\right)\left(f(t x)-\frac{m}{N} g(t y)\right) \geq 0, \quad t>0 ; x, y \in[0,1] .
$$

The expanded inequality

$$
\left(\frac{M}{n}+\frac{m}{N}\right) f(t x) g(t y) \geq f^{2}(t x)+\frac{m M}{n N} g^{2}(t y)
$$

when multiplied by the product of two different-parameter Saigo's hypergeometric kernels

$$
\frac{t^{-2 \beta}}{[\Gamma(\alpha)]^{2}}[(1-x)(1-y)]^{\alpha-1}{ }_{2} F_{1}\left[\begin{array}{c}
\alpha+\beta,-\eta \\
\alpha
\end{array} ; 1-x\right]{ }_{2} F_{1}\left[\begin{array}{c}
\alpha+\beta,-\xi \\
\alpha
\end{array} ; 1-y\right],
$$

and integrated with respect to $x$ and $y$ on the square $[0,1]^{2}$, becomes

$$
I_{0, t}^{\alpha, \beta, \eta}\left[f^{2}\right] I_{0, t}^{\alpha, \beta, \xi}[1]+\frac{m M}{n N} I_{0, t}^{\alpha, \beta, \eta}[1] I_{0, t}^{\alpha, \beta, \xi}\left[g^{2}\right] \leq\left(\frac{M}{n}+\frac{m}{N}\right) I_{0, t}^{\alpha, \beta, \eta}[f] I_{0, t}^{\alpha, \beta, \xi}[g] .
$$

It is enough to renormalize the last display by $I_{0, t}^{\alpha, \beta, \eta}[1] \cdot I_{0, t}^{\alpha, \beta, \xi}[1]$ to obtain (2.3).

For the allied RL and EK special cases of the Saigo-type Diaz-Metcalf inequality (2.3) we deduce the following forms:

$$
\begin{aligned}
& \mathscr{M}_{\mathrm{RL}}^{\eta}\left(f^{2}\right)+\frac{m M}{n N} \mathscr{M}_{\mathrm{RL}}^{\xi}\left(g^{2}\right) \leq\left(\frac{m}{N}+\frac{M}{n}\right) \mathscr{M}_{\mathrm{RL}}^{\eta}(f) \mathscr{M}_{\mathrm{RL}}^{\xi}(g), \\
& \mathscr{M}_{\mathrm{EK}}^{\eta}\left(f^{2}\right)+\frac{m M}{n N} \mathscr{M}_{\mathrm{EK}}^{\xi}\left(g^{2}\right) \leq\left(\frac{m}{N}+\frac{M}{n}\right) \mathscr{M}_{\mathrm{EK}}^{\eta}(f) \mathscr{M}_{\mathrm{EK}}^{\xi}(g),
\end{aligned}
$$

for all $\Re(\alpha), \eta>0$ and $t>0$.

Comparing the inequalities' right-hand-side expressions in Theorems 1 and 2 we see that the terms $\mathscr{M}_{\mathrm{S}}(f g) \equiv \mathscr{M}_{\mathrm{S}}^{\eta}(f g)$ and $\mathscr{M}_{\mathrm{S}}^{\eta}(f) \mathscr{M}_{\mathrm{S}}^{\xi}(g)$ differ even in the case $\eta=\xi$. To discuss this question we recall the Cebyšev integral inequality [7, p. 40]: If $f, g:[a, b] \rightarrow \mathbb{R}$ are integrable functions, both increasing or both decreasing, and $p:[a, b] \rightarrow \mathbb{R}_{+}$is an integrable function, then

$$
\int_{a}^{b} p(x) f(x) \mathrm{d} x \int_{a}^{b} p(x) g(x) \mathrm{d} x \leq \int_{a}^{b} p(x) \mathrm{d} x \int_{a}^{b} p(x) f(x) g(x) \mathrm{d} x .
$$

Assume that $\eta=\xi$ and both functions $f, g$ increase (decrease) simultaneously on $[0, t], t>0$, then choosing $p(x)=w_{\mathrm{S}}(x)$ (compare (1.11)), by the above listed Cebyšev integral inequality, we arrive at the classical Diaz-Metcalf inequality:

$$
\mathscr{M}_{\mathrm{S}}\left(f^{2}\right)+\frac{m M}{n N} \mathscr{M}_{\mathrm{S}}\left(g^{2}\right) \leq\left(\frac{m}{N}+\frac{M}{n}\right) \mathscr{M}_{\mathrm{S}}(f g),
$$

where, we transform the upper bound in (2.3). The same conclusions follow for the related RiemannLiouville and Erdélyi-Kober integral means. 


\section{On Pólya-Szegö inequality for Saigo type operator}

The present topic is strongly connected in view of the Remark 1 to Diaz-Metcalf inequality chapter's matter.

Theorem 3.1. Let $f, g$ be bounded functions on $\mathbb{R}_{+}$which satisfy (1.1). Then for all $\Re(\alpha)>$ $0, \beta<1, \eta>\beta-1$, and for all $t>0$ we have

$$
\frac{\mathscr{M}_{\mathrm{S}}\left(f^{2}\right) \mathscr{M}_{\mathrm{S}}\left(g^{2}\right)}{\left[\mathscr{M}_{\mathrm{S}}(f g)\right]^{2}} \leq \frac{1}{4}\left(\sqrt{\frac{M N}{m n}}+\sqrt{\frac{m n}{M N}}\right)^{2} .
$$

Proof. Following the standard proof of the Pólya-Szegő inequality, applying the Arithmetic meanGeometric mean inequality to the left-hand-side expression of (2.1) yields

$$
\left(\frac{m}{N}+\frac{M}{n}\right) \mathscr{M}_{\mathrm{S}}(f g) \geq \mathscr{M}_{\mathrm{S}}\left(f^{2}\right)+\frac{m M}{n N} \mathscr{M}_{\mathrm{S}}\left(g^{2}\right) \geq 2 \sqrt{\frac{m M}{n N} \mathscr{M}_{\mathrm{S}}\left(f^{2}\right) \mathscr{M}_{\mathrm{S}}\left(g^{2}\right)} ;
$$

obvious transformations of two utmost terms lead to the asserted inequality (3.1).

Q.E.D.

For the equality analysis in this and similar cases we refer to [8, p. 117].

The related Pólya-Szegó type inequalities are valid as well for the Riemann-Liouville and Erdélyi-Kober integral operators. Actually, we get these special cases of Theorem 3 choosing $\beta=-\alpha$, and $\beta=0$ respectively.

The corresponding counterpart of the Pólya-Szegö inequalities (1.5) and (1.8) considering two different weighted Saigo integral means which contain arbitrary independent scale parameters $\eta$ and $\xi$, reads as follows.

Theorem 3.2. Let $f, g$ be positive integrable functions on $\mathbb{R}_{+}$. When $f, g$ satisfy (1.1) then for all $\Re(\alpha)>0, \beta<1, \min \{\eta, \xi\}>\beta-1$ we have

$$
\frac{\mathscr{M}_{\mathrm{S}}^{\eta}\left(f^{2}\right) \mathscr{M}_{\mathrm{S}}^{\xi}\left(g^{2}\right)}{\left[\mathscr{M}_{\mathrm{S}}^{\eta}(f) \mathscr{M}_{\mathrm{S}}^{\xi}(g)\right]^{2}} \leq \frac{1}{4}\left(\sqrt{\frac{M N}{m n}}+\sqrt{\frac{m n}{M N}}\right)^{2} .
$$

Proof. By virtue of the Arithmetic mean-Geometric mean inequality we transform the extended two parameter variant Diaz-Metcalf inequality (2.3) into

$$
\left(\frac{m}{N}+\frac{M}{n}\right) \mathscr{M}_{\mathrm{S}}^{\eta}(f) \mathscr{M}_{\mathrm{S}}^{\xi}(g) \geq 2 \sqrt{\frac{m M}{n N} \mathscr{M}_{\mathrm{S}}^{\eta}\left(f^{2}\right) \mathscr{M}_{\mathrm{S}}^{\xi}\left(g^{2}\right)} .
$$

Now, obvious transformations result in the statement (3.2).

Q.E.D.

We can also state the related Riemann-Liouville and Erdélyi-Kober variants of the last theorem, by taking $\beta=-\alpha$, and $\beta=0$ respectively in Theorem 4 , leaving these routine moves to the interested reader.

Moreover using $f \equiv g$ on the whole range of the functions, we deduce the following interesting result. 
Corollary 3.3. Let $f$ be integrable functions on $\mathbb{R}_{+}$which satisfies (1.1). Then for all $\Re(\alpha)>$ $0, \beta<1, \min \{\eta, \xi\}>\beta-1$ we have

$$
\frac{\mathscr{M}_{\mathrm{S}}^{\eta}\left(f^{2}\right) \mathscr{M}_{\mathrm{S}}^{\xi}\left(f^{2}\right)}{\left[\mathscr{M}_{\mathrm{S}}^{\eta}(f) \mathscr{M}_{\mathrm{S}}^{\xi}(f)\right]^{2}} \leq \frac{1}{4}\left(\frac{M}{m}+\frac{m}{M}\right)^{2} .
$$

Finally, by $n=N$ (or by the equivalent specification $g \equiv 1$ ), we get

Corollary 3.4. Let $f$ be integrable functions on $\mathbb{R}_{+}$which satisfies (1.1). Then for all $\Re(\alpha)>$ $0, \beta<1, \eta>\beta-1$ we have

$$
\mathscr{M}_{\mathrm{S}}^{\eta}\left(f^{2}\right) \leq \frac{1}{4}\left(\frac{M}{m}+\frac{m}{M}\right)^{2}\left[\mathscr{M}_{\mathrm{S}}^{\eta}(f)\right]^{2} .
$$

\section{On Rennie and Schweitzer-type inequalities}

The derived results have a set of consequent inequalities known under different names, like Kantorovič, Greub-Rheinboldt, Renie, Cassels, Schweitzer etc. inequalities, see e.g. [3, 5, 7, 8, 10, 13]. We presnt some of them in our Saigo integral operator setting.

Firstly, we present Rennie-type single-input-function inequalities whose origins can be found in [10], also see [7, 8]. The resulting inequalities are of the Rennie-type, see

Theorem 4.1. Let $F \in L_{w_{\mathrm{S}}}^{1}[0, t]$ and $0<m \leq F(x) \leq M<\infty, x \in[0, t]$. Then for all $\Re\{\alpha\}>0, \beta<1, \eta>\beta-1$, and for all $t>0$ we have

$$
\mathscr{M}_{\mathrm{S}}(F)+m M \mathscr{M}_{\mathrm{S}}(1 / F) \leq m+M \quad \Re(t)>0 .
$$

Moreover, for all $\Re\{\alpha\}>0, \beta<1 ; \eta, \xi \in \mathbb{R}$ such that $\min \{\eta, \xi\}>\beta-1$, and for all $t>0$ we have

$$
\mathscr{M}_{\mathrm{S}}^{\eta}(F)+m M \mathscr{M}_{\mathrm{S}}^{\xi}(1 / F) \leq m+M \mathscr{M}_{\mathrm{S}}^{\eta}(F) \mathscr{M}_{\mathrm{S}}^{\xi}(1 / F) \quad \Re(t)>0 .
$$

Proof. As $F$ obeys $0<m \leq F(x) \leq M<\infty$, we can write

$$
\left(\frac{M}{F(t x)}-1\right)(F(t x)-m) \geq 0 .
$$

The resulting expression

$$
F(t x)+m M \frac{1}{F(t x)} \leq m+M
$$

multiplied by the Saigo's hypergeometric kernel $K_{\eta}(x)$ and integrated with respect to $x \in[0,1]$ gives via the normalization by $I_{0, t}^{\alpha, \beta, \eta}[1]$ the inequality (4.1).

Moreover, considering

$$
\left(\frac{M}{F(t y)}-1\right)(F(t x)-m) \geq 0,
$$

we get

$$
F(t x)+m M \frac{1}{F(t y)} \leq m+M \frac{F(t x)}{F(t y)} .
$$

The desired inequality (4.2) we obtain by multiplying the last display with $K_{\eta}(x) K_{\xi}(y)$, then integrating it on $(x, y) \in[0,1]^{2}$ and normalizing by $I_{0, t}^{\alpha, \beta, \eta}[1] I_{0, t}^{\alpha, \beta, \xi}[1]$.

Q.E.D. 
Finally, applying the Arithmetic mean-Geometric mean inequality to the left-hand side expression in (4.1), we easily complete the proof of the so-called Schweitzer-type inequality; the origins of which have been reported in [13], consult also [7, 8].

Theorem 4.2. Let $F \in L_{w_{\mathrm{S}}}^{1}[0, t]$ and $0<m \leq F(x) \leq M<\infty, x \in[0, t]$. Then for all $\Re\{\alpha\}>0, \beta<1, \eta>\beta-1$, and for all $t>0$ we have

$$
\mathscr{M}_{\mathrm{S}}(F) \mathscr{M}_{\mathrm{S}}(1 / F) \leq \frac{1}{4}\left(\sqrt{\frac{M}{m}}+\sqrt{\frac{m}{M}}\right)^{2} \quad \Re(t)>0 .
$$

Remark 4.3. Let us remark that the same kind of procedure applied to the left-hand-side terms in (4.2) yields

$$
\left[\sqrt{M}\left(\mathscr{M}_{\mathrm{S}}(F) \mathscr{M}_{\mathrm{S}}(1 / F)\right)^{1 / 2}-\sqrt{m}\right]^{2} \geq 0,
$$

which is obviously not of any further interest.

Also it is worth to mention that a comprehensive equality analysis for Diaz-Metcalf inequality can be found in [2,3], while further equivalence results have been established between Rennie's and the celebrated Diaz-Metcalf inequalities in [2].

\section{References}

[1] A. Anber and Z. Dahmani, New integral results using Pólya-Szegő inequality, Acta Comment. Univ. Tartu. Math. 17 (2) (2013), 171-178.

[2] J. B. Diaz, A. J. Goldman and F. T. Metcalf, Equivalence of certain inequalities complementing those of Cauchy-Schwarz and Hölder, J. Res. Nat. Bur. Standards Sect. B 68B (1964), 147149.

[3] J. B. Diaz and F. T. Metcalf, Stronger forms of a class of inequalities of G. Pólya - G. Szegö and L.V.Kantorovich, Bull. Amer. Math. Soc. 69(1963), 415-418.

[4] I. S. Gradshteyn and I. M. Ryzhik, Table of Integrals, Series and Products, Sixth Edition, Academic Press, San Diego · San Francisco · New York · Boston · London Sidney · Tokyo, 2000.

[5] W. Greub and W. Rheinboldt, On a generalization of an inequality of L. V. Kantorovich, Proc. Amer. Math. Soc. 10 (1959), 407-415.

[6] D. Jankov and T. K. Pogány, Andreev-Korkin identity, Saigo fractional integration operator and $\operatorname{Lip}_{L}(\alpha)$ functions, J. Math. Phys. Anal. Geometry 8 (2012), 144-157.

[7] D.S. Mitrinović, Analytic Inequalities, Graḍevinska knjiga, Beograd, 1970. (Serbian)

[8] T. K. Pogány, New (probabilistic) derivation of Diaz-Metcalf and Pólya-Szegő inequalities and consequences, Teor. Probab. Mat. Statist. 70 (2005), 113-122. 
[9] G. Pólya and G. Szegő, Aufgaben und Lehrsätze aus der Analysis. Band 1, Die Grundlehren der mathematischen Wissenschaften 19, J. Springer, Berlin, 1925.

[10] B. C. Rennie, On a class of inequalities, J. Austral. Math. Soc. 3(1963), 442-448.

[11] M. Saigo, A remark on integral operators involving Gauss's hypergeometric functions, Math. Rep. Kyushu Univ. 11(2) (1977/1978), 135-143.

[12] R. K. Saxena, J. Ram, J. Daiya and T. K. Pogány, Inequalities associated with Čebyšev functional for Saigo fractional integration operator, Integral Transforms Spec. Funct. 22 (9) (2011), 671-680.

[13] P. Schweitzer, An inequality concerning the arithmetic mean, Math. Phys. Lapok 23(1914), 257-261. (in Hungarian) 